\title{
GENERATING RANDOM ELEMENTS OF FINITE DISTRIBUTIVE LATTICES
}

\author{
JAMES PROPP
}

\begin{abstract}
This survey article describes a method for choosing uniformly at random from any finite set whose objects can be viewed as constituting a distributive lattice. The method is based on ideas of the author and David Wilson for using "coupling from the past" to remove initialization bias from Monte Carlo randomization. The article describes several applications to specific kinds of combinatorial objects such as tilings, constrained lattice paths, and alternating-sign matrices.
\end{abstract}

\section{This article is dedicated to Herbert Wilf in honor of his sixty-fifth birthday.}

\section{INTRODUCTION}

Herb Wilf, in addition to having done important work on problems related to counting combinatorial objects, has also done pioneering research on algorithms for generating combinatorial objects "at random" (that is, generating an element of a finite combinatorial set so that each element has the same probability of being generated as any other); see

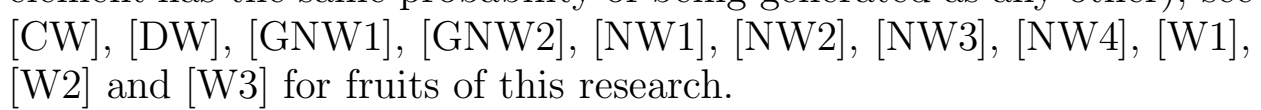

This survey article describes a recent advance in the area of random generation, with applications to plane partitions, domino tilings, alternating sign matrices, and many other sorts of combinatorial objects. The algorithm is of the "random walk" or "Monte Carlo" variety, but unlike many such algorithms it does not have any initialization bias. The heart of the algorithm is the method of coupling from the past explored by David Wilson and myself in a joint article [PW]. For the sake of readability and motivation, I will start by focusing on the application of our method to plane partitions.

Date: March 3, 1997.

During the conduct of the research that led to this article, the author was supported by NSA grant MDA904-92-H-3060, NSF grant DMS 9206374 and a grant from the MIT Class of 1922. 
A beautiful formula of MacMahon [M] says that the number of plane partitions of $n$ with at most $a$ rows, at most $b$ columns, and no part exceeding $c$ (hereafter to be called "( $a, b, c)$-partitions") is given by

$$
\prod_{i=0}^{a-1} \prod_{j=0}^{b-1} \prod_{k=0}^{c-1} \frac{i+j+k+2}{i+j+k+1}
$$

(see [A] and [S] for definitions of ordinary partitions and plane partitions, and section 2 of [CLP] for a fairly simple self-contained proof of MacMahon's formula).

Note that in the case $c=1$, a plane partition with no part exceeding 1 can be read as the Ferrers diagram of an ordinary partition, and MacMahon's formula devolves into the assertion that the number of ordinary partitions of $n$ with at most $a$ parts and no part exceeding $b$ is given by the binomial coefficient $\frac{(a+b) !}{a ! b !}$. Indeed, it is easy to see that such partitions correspond to lattice paths of length $a+b$ joining $(0, a)$ to $(b, 0)$, or equivalently, combinations of $a+b$ elements taken $a$ at a time. In view of these correspondences, it is easy to generate a random $(a, b, 1)$-partition.

Just as a lattice path in the $a$ by $b$ rectangle is a 1-complex (made up of edges in a 2-dimensional grid) with prescribed boundary (namely the pair of points $(0, a)$ and $(b, 0))$, an $(a, b, c)$-partition corresponds to a 2-complex (made up of square 2-cells in a 3-dimensional grid) whose boundary is a particular non-planar hexagon (namely the one that goes from $(a, 0,0)$ to $(a, b, 0)$ to $(0, b, 0)$ to $(0, b, c)$ to $(0,0, c)$ to $(a, 0, c)$ to $(a, 0,0)$ in cyclic order). In the former case, one requires that the lattice path should have (minimal) length $a+b$; in the latter case, one requires that the surface spanning the hexagon should have (minimal) area $a b+b c+a c$.

In this paper I will describe an algorithm for generating a random $(a, b, c)$-partition with $a, b, c$ arbitrary. This algorithm was used to generate Figure 1, which shows a random $(32,32,32)$-partition, or rather the spanning surface that it determines, viewed from a point on the ray $x=y=z>0$; the three different orientations of grid-squares in 3space are seen in projection as three different orientations of rhombuses in 2-space. It should be stressed that the size of the plane partition (that is, the sum of the parts) was not specified in advance; it is a random variable with expected value $(32)^{3} / 2$.

Note the non-homogeneity of the picture: there is an approximately circular central region in which rhombuses of different orientations are mixed together, surrounded by six regions in which rhombuses of a single orientation predominate. As is shown in [CLP], in a certain 
strong probabilistic sense the boundary of the central region does indeed converge to a perfect circle when $n$ is large. This fact was first conjectured on the basis of pictures like Figure 1, and all known proofs depend on steps whose clearest motivation comes from "knowing the answer in advance". Thus we see that there are phenomena pertaining to random $(a, b, c)$-partitions that would not have been easy to divine by pure theory, and that an algorithm for generating such plane partitions randomly can be a valuable tool for discovering and investigating such phenomena experimentally.

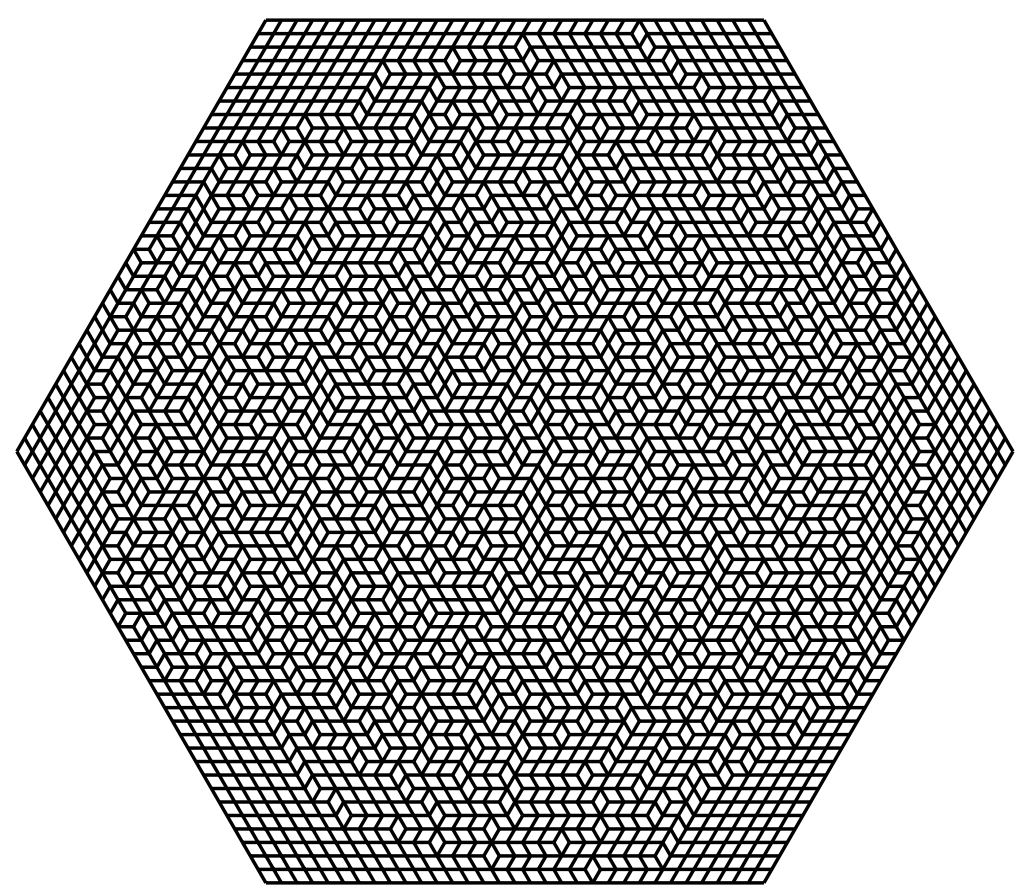

A random lozenge tiling of a $32,32,32$ hexagon.

In part 2 of this paper, I will show that this problem, along with several others, is a special case of the problem of choosing a random element of a finite distributive lattice. In part 3, I will describe an algorithm that allows one to solve this problem. Like the well-known folk-algorithm for generating a random element of a 3-element set via independent tosses of an unbiased coin, this algorithm runs in finite expected time, even though it can take arbitrarily long to return an answer. I conclude in part 4 with some open questions. 


\section{Distributive LATTICES}

The 3-dimensional Ferrers diagram associated with an $(a, b, c)$-partition is just an order ideal of the poset obtained as the product of chains of cardinalities $a, b$ and $c$. Writing such chains as $\mathbf{a}, \mathbf{b}$ and $\mathbf{c}$, respectively, and using $J(\cdot)$ to represent the lattice of order ideals of a finite poset (ordered by inclusion), we see that what we are effectively trying to do is generate a random element of the distributive lattice $J(\mathbf{a} \times \mathbf{b} \times \mathbf{c})$. (See $[\mathrm{S}]$ for definitions of posets, order ideals, lattices, and distributive lattices, and for the fact that every finite distributive lattice can be represented as $J(P)$ for some finite poset $P$.)

Here is another way to view this ordering. Consider a hexagon whose internal angles all measure 60 degrees and whose sides in cyclic order have lengths $a, b, c, a, b, c$, respectively, and dissect it into $2 a b+2 a c+2 b c$ unit equilateral triangles. Create a graph $G$ whose vertices correspond to these triangles, where two vertices of $G$ are adjacent if and only if the corresponding triangles in the dissection share an edge. ( $G$ is called a "honeycomb graph" for obvious reasons.) Then each $(a, b, c)$-partition corresponds to a tiling of the aforementioned hexagon by rhombuses of side length 1 (each composed of two equilateral triangles joined edge to edge), and each of these tilings in turn corresponds to a perfect matching of $G$, that is, to a subset of the edges of $G$ in which each vertex of $G$ appears just once. It is shown in $[\mathrm{P}]$ (which was inspired by earlier work of Conway and Lagarias [CL] and Thurston [T] that the perfect matchings of any bipartite planar graph can be given the structure of a distributive lattice. In the case of the honeycomb-graph $G$ described above, one obtains the same distributive lattice structure $J(\mathbf{a} \times \mathbf{b} \times \mathbf{c})$ as before. If instead one allows graphs $G$ that are subgraphs of a square grid, one is able to put a distributive lattice structure on the set of all tilings of a connected subset of the square grid by dominos. (These special cases are discussed in [T].)

More generally (again see $[\mathrm{P}]$ ), if $G$ ss any bipartite planar graph, and $d(\cdot)$ is any function from the vertex set of $G$ to the non-negative integers, then the set of $d$-factors of $G$ (defined as the set of subgraphs of $G$ in which vertex $v$ has degree $d(v)$ for all vertices $v$ ) can be given the structure of a distributive lattice (outside of the trivial case in which no $d$-factors exist).

Another class of examples of distributive lattices in combinatorics comes from constrained lattice paths. For instance, lattice paths of length $2 n$ that go from $(0, n)$ to $(n, 0)$ without straying outside of the triangle with vertices $(0,0),(n, 0)$ and $(n, 0)$ are a standard incarnation 
of the "Catalan objects". More generally, we can consider all minimallength lattice paths that go from one fixed lattice point to another without straying outside some fixed convex region. Assuming that the set of such lattice paths is non-empty, it is easy to show that the set of all such lattice paths is a distributive lattice, with the ordering being defined by inclusion of the associated Ferrers diagrams. One can also extend this approach to the lattice paths enumerated by trinomial coefficients (with step-vectors $(1,1),(1,0)$, and $(1,-1)$ ).

A further example is provided by the alternating sign matrices introduced by Mills, Robbins, and Rumsey [MRR]. One can transform each $n$ by $n$ alternating sign matrix into an $n+1$ by $n+1$ array of numbers in such a fashion that if one takes the component-wise minimum (or component-wise maximum) of any two such arrays, one gets another array associated with an $n$ by $n$ alternating sign matrix. In this fashion, the set of alternating sign matrices of fixed size becomes a distributive lattice. (See [EKLP] for a more detailed discussion of this.)

A final situation I will mention in which a non-obvious distributive lattice structure exists is the set of independent sets in a bipartite graph $G$. (This lattice structure has been noticed in the literature several times independently.) Here is the way to see it: If we color the vertices of $G$ black and white and write every independent set $S$ as $S_{B} \cup S_{W}$ where the vertices in $S_{B}$ and $S_{W}$ are black and white respectively, then we may define the meet and join of $S_{B} \cup S_{W}$ and $T_{B} \cup T_{W}$ as $\left(S_{B} \cup T_{B}\right) \cup\left(S_{W} \cap T_{W}\right)$ and $\left(S_{B} \cap T_{B}\right) \cup\left(S_{W} \cup T_{W}\right)$, respectively.

We recall here that every finite distributive lattice admits a unique representation (up to isomorphism) as $J(P)$, for $P$ some finite poset (which in fact is easily constructible from the lattice as the sub-poset of join-irreducibles). In each of the cases described above, the bijection between the combinatorial objects described and order ideals of an associated poset $P$ is easily implemented on a computer. Thus, if we can solve the problem of generating a random element of $J(P)$ for an arbitrary finite poset $P$, we will have solved the problem of generating random $d$-factors of bipartite planar graphs, the problem of generating random independent sets in a general bipartite graph, and many other problems as well.

\section{ThE ALGORITHM}

In this section I describe a Monte Carlo approach to sampling from the uniform distribution on the set $J(P)$, using a Markov chain whose state are the elements of $J(P)$ and whose steady state distribution is the uniform distribution on $J(P)$. If one merely simulated this Markov 
chain for a large but finite number of steps in the ordinary way, one would have a distribution that was close to the steady-state (uniform) distribution, but there would be some residual error ("initialization bias"). Later in this section I will explain how one can get rid of this bias by effectively simulating the Markov chain for infinitely many steps, from time minus infinity to time zero (the method of coupling from the past).

It is natural to make $J(P)$ into a graph $H$ by declaring two order ideals to be adjacent iff their symmetric difference consists of exactly one element of $P$. It is easy to show that this graph is connected. Moreover, we can associate with each element $x$ of $P$ a randomization move that preserves the uniform distribution on $J(P)$ (the probability distribution that assigns each order ideal probability $1 /|J(P)|)$ : Given an order ideal $I$, toss a fair coin, and if the coin comes up heads (resp. tails), replace $I$ by $I \cup\{x\}$ (resp., $I \backslash\{x\}$ ), unless the resulting subset of $P$ is not an order ideal, in which case leave $I$ alone.

If one performs an infinite sequence of such randomization moves in which each new randomization site $x$ is chosen independently from the uniform distribution on $P$ (or more generally from any probability distribution that assigns positive probability to each element of $P$ ), then this process is simply a stationary Markov chain whose state space is the set of order ideals of $P$; standard ideas from the theory of Markov chains guarantee that the probability of any particular order ideal being the current order ideal converges to $1 /|J(P)|$ as time goes to infinity.

This Markov chain gives us a way, in principle, of generating a random element of $J(P)$ that is as close to unbiased as we like (i.e., whose governing distribution is as close to uniform as we like). However, in the absence of estimates of the mixing time of the Markov chain, it is not clear for how long a time the chain must be run in order to drive the bias below some predetermined amount deemed acceptable. Moreover, we seek a way of generating samples that has no bias when truly random bits are used.

Fortunately, there is a way around this problem. The Markov chain that we have described is monotone in the sense that if we were to run two instantiations of it in parallel, using the same randomization sites and the same coin-tosses in both runs but starting from different initial order ideals $I_{1}$ and $I_{2}$, then, provided $I_{1} \supseteq I_{2}$, the order ideals $I_{1}^{\prime}$ and $I_{2}^{\prime}$ that result after $n$ steps of joint randomization must satisfy the relation $I_{1}^{\prime} \supseteq I_{2}^{\prime}$. In particular, if we had chosen $I_{1}$ and $I_{2}$ to be the empty order ideal $\hat{0}=\emptyset$ and the full order ideal $\hat{1}=P$, respectively, and if we find after $n$ steps that $I_{1}^{\prime}$ and $I_{2}^{\prime}$ are equal (call their common value $I^{\prime}$ ), then every run of the Markov chain for $n$ steps using those 
randomization sites and coin-tosses will put us in state $I^{\prime}$, regardless of the initial state $I$; all such histories are "squeezed" between the $\hat{0}$ history and the $\hat{1}$-history, and since these coalesce over the course of the simulation, so must all histories.

It turns out that for most of the examples that arise in practice, this kind of coalescence occurs fairly quickly. This gives us a way of doing a kind of "backwards simulation" of the Markov chain that effectively lets us run the chain for a huge number of steps without (usually) having to perform anywhere near the number of steps required in a straightforward simulation. Say, for instance, that we want to run the Markov chain on the state-space $J(P)$ for one million steps, starting from initial state $I^{*}$, using random updates from time $-1,000,000$ to time 0 (we will see shortly why it is convenient to index time in this way). Suppose that our Markov chain is sufficiently rapidly mixing that over the course of a thousand steps, it's fairly likely for the initial states $\hat{0}$ and $\hat{1}$ to lead to the same final state when evolved in tandem. If we simulate the Markov chain from time -1000 to time 0, using both $\hat{0}$ and $\hat{1}$ as initial states (let us call this "phase one" of our backwards simulation), and we indeed find that both histories coalesce at some state $I^{\prime}$ at time 0 , then we do not need to run the full simulation from one million steps in the past, for we can already be sure that such a simulation would have given us $I^{\prime}$ as our sample. Indeed, in this case we do not even have to choose what the randomization sites and coin-tosses before time -1000 are, since they do not enter into the simulation. In the unlikely event that the two histories that were started at time -1000 do not coalesce by time 0 , then we could go back and do an honest simulation for a million steps, starting from $I^{*}$ at time $-1,000,000$ (let us call this "phase two"). In this way we can simulate the behavior of a million-step random walk using (most of the time) only about two thousand simulation steps. Note however that if one wishes to avoid introducing bias into one's sample, it is crucial that one use the same randomization sites and coin-tosses from time -1000 onward during the long, "honest" run (phase two) as one did during the short preliminary run (phase one).

If one wants to simulate a billion steps of random walk, one can modify phase two of the preceding algorithm by running the million-step simulation (in the rare case where one thousand steps do not suffice) using $\hat{0}$ and $\hat{1}$ as starting states, rather than $I^{*}$. Only in the incredibly rare case where this million-step simulation fails to coalesce by time zero would one need to resort to a billion-step simulation starting 
from $I^{*}$ ("phase three"), being careful once more to use the alreadydetermined randomization sites and coin-tosses from time $-1,000,000$ to time 0 .

Note that the numbers one million and one billion enter into these procedures in the form of bounds on how far back into the past one is willing to go before one "honestly" uses $I^{*}$ as the starting state rather than trying to be clever by starting from $\hat{0}$ and $\hat{1}$ and hoping that they will coalesce by time 0 . Suppose that one removes this upper bound on how far into the past one is willing to go: if coalescence fails, one goes back into the past 1000 times as far as one just did and tries again. Then one can show that with probability 1 the desired coalescence will eventually occur (where our notion of "eventuality" goes backward in time rather than forward), and that the sample returned is effectively a sample "generated by a run of length infinity" - that is, an absolutely unbiased sample. This is David Wilson's method of coupling from the past.

It cannot be overemphasized that the idea of progressing backwards into the past is an indispensable feature of the algorithm. In particular, if one were simply to run the Markov chain forwards from initial states $\hat{0}$ and $\hat{1}$ in tandem until the histories coalesced and then to output as one's sample the coalescent state, one would in general get a biased sample. In contrast, samples generated via coupling from the past are entirely free of bias, to the extent that the coin-tosses used are random.

The version of the algorithm discussed above can be improved upon; for instance, it is much better to go progressively $1,2,4,8, \ldots$ steps into the past rather than $10^{3}, 10^{6}, 10^{9}, \ldots$ steps. Moreover, the algorithm can be generalized so as to apply to interesting situations in statistical mechanics where the desired distribution is not uniform, but is the Boltzmann distribution for some non-constant energy function on the configuration space. All of these developments are described more fully in $[\mathrm{PW}]$

Here, we content ourselves with showing that the expected running time is finite, in the case where there exists a finite $L$ and a positive $\epsilon$ such that over the course of any time interval of length $L$ in the simulation, the conditional probability of coalescence occurring, given initial states $\hat{0}$ and $\hat{1}$, is at least $\epsilon$. (This holds for all the interesting applications; in each case, it suffices to find an $L$ such that the probability of going from $\hat{0}$ to $\hat{1}$ in $L$ steps, conditional upon starting in state $\hat{0}$, is positive, though the $L$ one gets from this approach is much, much larger than the typical time-scale over which coalescence occurs.) To prove the claim, note that over the course of $k L$ consecutive steps, divided into $k$ blocks of length $L$, the only way in which coalescence could 
fail to occur is if all $k$ blocks are "non-coalescing"; yet independence of the coin-tosses tells us that this is an event of probability at most $(1-\epsilon)^{n}$. As $n$ gets large, this probability shrinks to zero exponentially, implying our claim on the finiteness of expected running time.

The algorithm described above is not always efficient; indeed, in $[\mathrm{PW}]$ Wilson and I describe a case in which the modified Monte Carlo algorithm takes exponentially long, even though a direct algorithm for constructing a random order ideal is quite easy to fashion (see the Cautionary Note at the end of section 3.2 in [PW]) However, as a practical matter the modified Monte Carlo algorithm is quite efficient for many sorts of combinatorial objects that arise naturally, such as those mentioned in Section 2.

Three final points on implementation deserve note.

First, the algorithm remains valid if instead of choosing randomization sites in accordance with some fixed distribution on $P$ one chooses randomization sites according to some other scheme, provided that two conditions are satisfied: the choice of randomization sites should not be affected by the outcomes of the coin flips, and it must be the case that with probability 1 each element of $P$ gets chosen as a randomization site infinitely often. Under these hypotheses, the (non-stationary) Markov chain on $J(P)$ has the uniform distribution as its unique steady-state measure, and the method of coupling from the past will get you there with a finite amount of simulation (with probability 1). For instance, one may rotate among all the elements of $P$ in some fixed order, rather than choosing the randomization sites randomly. In this way one reduces the amount of information that the algorithm needs to save.

Second, if one is using pseudo-random bits given by some trusted pseudo-random number generator (as I imagine most users of this algorithm will do), then, though it is often necessary to reuse bits, it is not necessary to save them all. One can for instance save only seeds that will enable one to re-create the formerly used bits when they are needed again.

Third, it should be borne in mind that the output of the Monte Carlo method is likely to be somewhat correlated to its running-time. If one were to use the method to generate $N$ samples, where $N$ was determined on-the-fly rather than chosen in advance, then one might be contaminating one's samples with bias. The scrupulous investigator may therefore wish to commit to a certain value of $N$ ahead of time, based on a preliminary investigation of how long it is likely to take to run the procedure $N$ times. 


\section{Open problems}

Another sort of scheme for randomly generating an order ideal of a finite poset $P$ is the recursive approach in which one decides whether or not to include $x$ in the random order ideal by tossing a coin whose bias corresponds to the ratio of the cardinalities of two distributive lattices, namely, the lattice of order ideals of $P$ containing $x$ and the lattice of order ideals of $P$ not containing $x$. This method (like coupling from the past) will not always be fast, since the problem of counting antichains (and hence, equivalently, order ideals) in a finite partially ordered set is \#P-complete (see [PB] . Yet another approach would be one along the lines of recent work of Flajolet, Zimmermann, and van Cutsem (see [FZC1] and [FZC2]), who have already created Maple software that (suitably instructed) can create random plane partitions of the sort shown in Figure 1. Which way is "best"? (Or rather: are there features of a lattice that might dictate when one or another of these approaches will do best?)

In the case of planar graphs $G$ that are not bipartite, it is still possible to generate random perfect matchings of $G$ efficiently: The method of Kasteleyn [[K] allows one to write the number of perfect matchings as a Pfaffian. By calculating the ratio of two such Pfaffians, one can determine the exact proportion of matchings of $G$ that contain a given edge, and one can accordingly make an unbiased decision as to whether or not to include this edge in the matching. Applying this recursively, one can generate a random matching of $G$. Surprisingly, Wilson [Wi] showed that it is possible to randomly generate perfect matchings within a constant multiple of the time needed to compute just one Pfaffian; using sparse linear algebra, this time is $O\left(n^{3 / 2}\right)$ arithmetic operations, or $O\left(n^{5 / 2} \log ^{2} n \log \log n\right)$ bit operations. In the case where $G$ is bipartite, a specialized version of the Pfaffian method (the permanent-determinant method) applies in much the same way; but in this situation one can also apply the Markov chain algorithm described in this paper. Can the method of this paper be extended to apply in the non-bipartite case?

If one uses a biased coin in place of a fair one, one can devise an algorithm in which the probability of a particular order ideal $I$ being generated is proportional to $q^{|I|}$, where $|I|$ is the cardinality of $I$ and $q$ is any positive real number. That is, we can generate a random element of a distributive lattice so that all the elements of rank $k$ in the lattice have individual probability proportional to $q^{k}$ of being picked. We might try to choose $q$ so as to maximize the collective probability of the elements of rank $k$, but even using this optimal $q$ we might have 
to run the biased procedure many times before we obtain a sample in rank $k$. Note, however, that the resulting sample will be governed by a uniform distribution on the $k$ th rank of the lattice. Is there an efficient procedure for selecting an element in a particular rank of a finite distributive lattice? Note that work of Flajolet, Zimmermann and van Cutsem $[\mathrm{FZC2}]$ provides a solution in some cases.

Lastly: To what extent can the method of coupling from the past be extended to sampling from more general partially ordered sets, such as modular lattices or lattices in general? For an example of an application of the method to a special kind of non-distributive lattice, see the paragraphs in subsection 3.3 of $[\mathrm{PW}]$ that treat permutations.

I thank the referee for many helpful comments.

\section{REFERENCES}

[A] G. Andrews, The Theory of Partitions, Addison-Wesley, 1976.

[CW] E. Calabi and H. Wilf, The sequential and random selection of subspaces over a finite field, J. Combinatorial Theory 22 (1977), 107-109.

[CL] H. Conway and J. Lagarias, Tilings with polyominoes and combinatorial group theory, J. Combin. Theory A 53 (1990), 183-208.

[CLP] H. Cohn, M. Larsen and J. Propp, The Shape of a Typical Boxed Plane Partition, preprint, 1996.

[DW] J. Dixon and H. Wilf, The random selection of unlabeled graphs, J. Algorithms 4 (1983), 205-213.

[EKLP] N. Elkies, G. Kuperberg, M. Larsen and J. Propp, Alternating Sign Matrices and Domino Tilings, J. Alg Combinatorics 1 (1992), 111-132 and 219-234.

[FZC1] P. Flajolet, P. Zimmermann and B. van Cutsem, A calculus for the random generation of labelled combinatorial structures, Theoret. Comput. Sci. 132 (1994), 1-35.

[FZC2] P. Flajolet, P. Zimmermann and B. van Cutsem, A calculus of random generation: unlabelled structures, preprint, 1996.

[GNW1] C. Greene, H. Nijenhuis and H. Wilf, A probabilistic proof of a formula for the number of Young Tableaux of a given shape, Adv. in Math. 31 (1979), 104-109.

[GNW2] C. Greene, A. Nijenhuis and H. Wilf, Another probabilistic method in the theory of Young Tableaux, J. Combinatorial Theory 37 (1984), 127-135.

[K] P.W. Kasteleyn, The statistics of dimers on a lattice, I. The number of dimer arrangements on a quadratic lattice, Physica 27, 1209-1225 (1961).

[M] P.A. MacMahon, Combinatory Analysis, Cambridge University Press, 1915-16 (reprinted by Chelsea Publishing Company, New York, 1960).

[MRR] W. Mills, D. Robbins, and H. Rumsey, Jr., Alternating sign matrices and descending plane partitions, J. Comb. Theory A 34 (1983), 340-359.

[NW1] A. Nijenhuis and H. Wilf, A method and two algorithms in the theory of partitions, J. Combinatorial Theory 18 (1975), 219-222. 
[NW2] A. Nijenhuis and H. Wilf, Combinatorial Algorithms, Academic Press, 1975.

[NW3] A. Nijenhuis and H. Wilf, Combinatorial Algorithms for Computers and Calculators (second edition of [NW2]), Academic Press, New York, 1978.

[NW4] A. Nijenhuis and H. Wilf, The enumeration of connected graphs and linked diagrams, J. Combinatorial Theory 27 (1979), 356-359.

[P] J. Propp, Lattice structure for orientations of graphs, preprint, 1993.

[PB] J. Provan and M. Ball, The complexity of counting cuts and of computing the probability that a graph is connected, SIAM J. Comput. 12 (1983), $777-788$

[PW] J. Propp and D. Wilson, Exact sampling with coupled Markov chains and applications to statistical mechanics, Random Structure and Algorithms, to appear.

[S] R. Stanley, Enumerative Combinatorics I, Wadsworth, 1986.

[T] W. Thurston, Conway's tiling groups, American Mathematical Monthly 97 (1990), 757-773.

[W1] H. Wilf, A unified setting for sequencing, ranking and random selection of combinatorial objects, Adv. in Math. 24 (1977), 281-291.

[W2] H. Wilf, A unified setting for selection algorithms, II, Annals of Discrete Mathematics 2: Algorithmic aspects of combinatorics, North Holland (1978), 135-148.

[W3] H. Wilf, The uniform selection of free trees, J. Algorithms 2 (1981), 204207.

[Wi] D. Wilson, Determinant algorithms for random planar structures, to appear in the published abstracts of the 1997 ACM-SIAM Symposium on Discrete Algorithms.

Department of Mathematics, Mit

E-mail address: propp@math.mit.edu 